\title{
Distributions and Euler systems in global function fields
}

\author{
by
}

Sunghan Bae (Taejon) and Linsheng Yin (Beijing)

0. Introduction. An ordinary distribution on $\mathbb{Q}$ with values in an abelian group $V$ (ordinary distribution from $\mathbb{Q} / \mathbb{Z}$ to $V$, in Kubert's terminology $[\mathrm{Ku}])$ is a map $\Theta: \mathbb{Q} / \mathbb{Z} \rightarrow V$ satisfying

$$
\Theta\left(\frac{n}{m}\right)=\sum_{i=0}^{d-1} \Theta\left(\frac{n+m i}{m d}\right)=\sum_{a \in \mathbb{Q} / \mathbb{Z}, d a=n / m} \Theta(a)
$$

for every positive integer $d$. Ordinary distributions on $\mathbb{Q}$ form a category. The image of the initial object of this category is called the universal ordinary distribution on $\mathbb{Q}$.

Let $\mu_{n}$ be the group of $n$th roots of unity, $\mu_{\infty}$ the group of all roots of unity, and $\mu_{\infty}^{*}=\mu_{\infty} \backslash\{1\}$. A circular distribution on $\mathbb{Q}$ is a Galois equivariant $\operatorname{map} \psi: \mu_{\infty}^{*} \rightarrow \overline{\mathbb{Q}}^{\times}$such that

$$
\psi(\varepsilon)=\prod_{\zeta^{d}=\varepsilon} \psi(\zeta)
$$

for any $\varepsilon \in \mu_{\infty}^{\times}$and any positive integer $d$. The most well-known example is $\psi(\zeta)=1-\zeta$. By an appropriate identification of $\mu_{\infty}$ with $\mathbb{Q} / \mathbb{Z}$ one can view a circular distribution as a certain (punctured) distribution with Galois equivariance.

Let $F$ be a finite abelian extension of $\mathbb{Q}$ and let $\mathbf{m}$ be the formal product of all prime numbers which split completely in $F$. An Euler system over $F$ is a collection $\left\{\xi_{n} \in F\left(\mu_{n}\right)^{\times}\right\}_{n \mid \mathbf{m}}$ such that

(0.3) $\xi_{n}$ is a global unit,

(0.4) $N_{F\left(\mu_{p n}\right) / F\left(\mu_{n}\right)} \xi_{p n}=\xi_{n}^{\mathrm{Fr}_{p}-1}$, where $\mathrm{Fr}_{p}$ is the Frobenius map at $p$,

(0.5) $\quad \xi_{p n} \equiv \xi_{n}$ modulo all prime ideals above $p$.

2000 Mathematics Subject Classification: Primary 11R58.

Research of S. Bae supported by KOSEF research grant R01-2006-000-10320-0 and F01-2006-000-10040-0.

Research of L. S. Yin supported by NSFC grant No. 10571097. 
Distributions and Euler systems play very important roles in the cyclotomic theory of $\mathbb{Q}$. The condition (0.4) may be interpreted as a certain restatement of (0.1) or (0.2). Thus one can expect close relations between distributions and Euler systems.

In fact, Anderson and Ouyang ([AO]) studied Euler systems via the group cohomology of the universal ordinary distribution on the rational number field. They constructed the universal Euler system $\left\{x_{m} \in \mathbb{U}_{m}\right\}_{m \mid \mathbf{m}}$, where $\mathbb{U}_{m}$ is the universal ordinary distribution of level $m$, and the universal Kolyvagin classes $\left\{c_{m} \in H^{0}\left(G_{m}, \mathbb{U}_{m} / M \mathbb{U}_{m}\right)\right\}_{m \mid \mathbf{m}}$ induced from the universal Euler system, where $G_{m}=\operatorname{Gal}\left(\mathbb{Q}\left(\zeta_{m}\right) / \mathbb{Q}\right)$ and $M$ is an odd positive integer. Then they showed that any Euler system and the corresponding Kolyvagin classes can be recovered from the universal ones by specialization and that the universal Kolyvagin classes satisfy a universal recursion independent of the choice of real abelian extension $F$ of $\mathbb{Q}$.

Moreover, Seo used Euler systems to attack Coleman's conjecture on the Galois module structure of the set of circular distributions. He then introduced a method of constructing Euler systems from circular distributions and gave some evidence indicating that the notions of circular distribution, Euler system and circular units are essentially the same ([S1, §4]). He also computed the torsion subgroups of the groups of circular distributions and of circular distributions with a certain congruence condition ([S2]).

The concept of distributions on general global fields was first introduced by Yin $[Y]$ generalizing the concept of distributions on $\mathbb{Q}$ of Kubert. It has proven to be very useful in studying the arithmetic of global fields, in particular, of global function fields. The cyclotomic theory of global function fields was well developed by many authors using sign normalized rank one Drinfeld modules. Using this theory, one can define circular distributions and Euler systems on global function fields, and study their properties analogous to those of circular distributions on the rational number field. In this article we consider the above questions studied by Anderson-Ouyang and Seo in the case of global function fields.

The rational number field and global function fields with a fixed place of degree one, which we will call infinity, have many properties in common. The most important are that they have only one infinite place and that the Galois groups of ray class fields over the Hilbert class field are direct products of inertia groups. In contrast to the rational number field, global function fields have nontrivial class groups. As a result the group cohomology of the universal ordinary distribution on a global function field is not well understood. But in the study of Euler systems, we only need to consider principal ideals. Thus we can also use the group cohomology method in the global function field case. 
Notations.

- $k$ : a global function field with the field of constants $\mathbb{F}_{q}$,

- $\infty$ : a fixed place of $k$ with degree one,

- $\mathbb{A}$ : the ring of functions of $k$ which are regular away from $\infty$.

We assume that $q>2$. Denote by $\mathfrak{e}$ the unit ideal of $\mathbb{A}$.

- $K_{\mathfrak{e}}=$ the Hilbert class field of $(k, \infty)$.

For an integral ideal $\mathfrak{m}$ of $\mathbb{A}$,

- $K_{\mathfrak{m}}:=$ the cyclotomic function field of the triple $(k, \mathfrak{m}, \infty)$ with conductor $\mathfrak{m}$ in the sense of Hayes [H1],

- $G_{\mathfrak{m}}:=\operatorname{Gal}\left(K_{\mathfrak{m}} / k\right), \quad H_{\mathfrak{m}}:=\operatorname{Gal}\left(K_{\mathfrak{m}} / K_{\mathfrak{e}}\right) \simeq(\mathbb{A} / \mathfrak{m})^{*}$,

- $K:=\bigcup_{\mathfrak{m}} K_{\mathfrak{m}}, \quad G:=\operatorname{Gal}(K / k)=\lim _{\mathfrak{m}} G_{\mathfrak{m}}, \quad H:=\operatorname{Gal}\left(K / K_{\mathfrak{e}}\right)=$ $\lim _{\mathfrak{m}} H_{\mathfrak{m}}$.

Let $\sigma_{\mathfrak{p}}$ be a generator of $H_{\mathfrak{p}}$.

- $N_{\mathfrak{p}}:=\sum_{i=0}^{q^{\operatorname{deg} \mathfrak{p}}-2} \sigma_{\mathfrak{p}}^{i} \quad$ and $\quad N_{\mathfrak{p}}^{\prime}:=\sum_{i=0}^{q^{\operatorname{deg} \mathfrak{p}}-2} i \sigma_{\mathfrak{p}}^{i}$.

Note that $N_{\mathfrak{p}}^{\prime}\left(\sigma_{\mathfrak{p}}-1\right)=\left(q^{\operatorname{deg} \mathfrak{p}}-1\right)-N_{\mathfrak{p}}$. For a square-free ideal $\mathfrak{m}$, let

- $N_{\mathfrak{m}}:=\prod_{\mathfrak{p} \mid \mathfrak{m}} N_{\mathfrak{p}}, \quad N_{\mathfrak{m}}^{\prime}:=\prod_{\mathfrak{p} \mid \mathfrak{m}} N_{\mathfrak{p}}^{\prime}$.

For each integral ideal $\mathfrak{g}$ of $\mathbb{A}$ let

- $n(\mathfrak{g}):=$ the number of distinct prime divisors of $\mathfrak{g}$,

- $o(\mathfrak{g}):=\sum_{\mathfrak{p}} \operatorname{ord}_{\mathfrak{p}}(\mathfrak{g})$.

\section{The universal ordinary distributions and Anderson's resolu- tion}

1.1. The universal ordinary distribution. We briefly recall some basic facts about the universal ordinary distribution on a global function field $k$. (See $[\mathrm{Y}],[\mathrm{BK}]$ for details. But note that our notations are slightly different from those of $[\mathrm{Y}]$.) Fix a sign function, i.e. a multiplicative function, sgn : $k_{\infty} \rightarrow \mathbb{F}_{\infty}=\mathbb{F}_{q}$ with $\operatorname{sgn}(0)=0$, where $\mathbb{F}_{\infty}$ is the residue field at $\infty$. We call $x \in k$ positive or monic if $\operatorname{sgn}(x)=1$. Let $T$ be the set of all nonzero fractional ideals of $\mathbb{A}$ and $T_{\mathfrak{e}}$ the set of all nonzero integral ideals of $\mathbb{A}$. For $\mathfrak{m} \in T_{\mathfrak{e}}$, let $T_{\mathfrak{m}}$ be the set of all nonzero fractional ideals which can be written as $\mathfrak{a m}^{-1}$ for some $\mathfrak{a} \in T_{\mathfrak{e}}$, and let $T_{\mathfrak{m}}^{\prime}$ be the set of all nonzero fractional ideals which can be written as $\mathfrak{a m}^{-1}$ for some $\mathfrak{a} \in T_{\mathfrak{e}}$ prime to $\mathfrak{m}$. We see that $T$ is the union of $T_{\mathfrak{m}}$ with $\mathfrak{m} \in T_{\mathfrak{e}}$, and $T_{\mathfrak{m}}$ is the disjoint union of $T_{\mathfrak{n}}^{\prime}$ for all ideals $\mathfrak{n} \mid \mathfrak{m}$.

We define an equivalence relation $\sim$ on $T$ : for $\mathfrak{u}$ and $\mathfrak{v}$ in $T, \mathfrak{u} \sim \mathfrak{v}$ if and only if $\mathfrak{v}=(1+x) \mathfrak{u}$ for some $x \in \mathfrak{u}^{-1}$ with $1+x$ positive. Let

$$
\bar{T}:=T / \sim, \quad \bar{T}_{\mathfrak{m}}:=T_{\mathfrak{m}} / \sim, \quad \bar{T}_{\mathfrak{m}}^{\prime}:=T_{\mathfrak{m}}^{\prime} / \sim .
$$


Note that, when $k=\mathbb{Q}, \bar{T}$ can be identified with $\mathbb{Q} / \mathbb{Z}$ and under this identification $\bar{T}_{(m)}$ becomes $(1 / m) \mathbb{Z} / \mathbb{Z}$, and $\bar{T}_{(m)}^{\prime}=((1 / m) \mathbb{Z} / \mathbb{Z})^{*}=\{a / m \in$ $(1 / m) \mathbb{Z} / \mathbb{Z}:(a, m)=1\}$.

For $\mathfrak{f} \in T$ we denote by $\{\mathfrak{f}\}$ the image of $\mathfrak{f}$ in $\bar{T}$. Then $T_{\mathfrak{e}}$ acts on $\bar{T}$ by $\mathfrak{n}\{\mathfrak{f}\}=\{\mathfrak{n} \mathfrak{f}\}$, and $G_{\mathfrak{m}}$ acts on $\bar{T}_{\mathfrak{m}}$ by $\sigma\{\mathfrak{f}\}=\{\mathfrak{a} \mathfrak{f}\}$ where $\sigma=\sigma_{\mathfrak{a}}$ is the element in $G_{\mathfrak{m}}$ associated to the ideal $\mathfrak{a}$ with $(\mathfrak{a}, \mathfrak{m})=\mathfrak{e}$ via the Artin map. Since $\bar{T}=\bigcup_{\mathfrak{m}} \bar{T}_{\mathfrak{m}}, \bar{T}$ becomes a $G$-set. In this way every $a \in \bar{T}$ can be uniquely written as $\sigma_{a}\left\{\mathfrak{d}_{a}^{-1}\right\}$ for some $\sigma_{a} \in G_{\mathfrak{d}_{a}}$, which can be thought as the analogue of $r / s$ with $r, s \in \mathbb{Z},(r, s)=1$ in the case of rational number field. In this sense, one may call $\sigma_{a}$ the numerator and $\mathfrak{d}_{a}$ the denominator of $a$. Or, one may view

$$
\mathbb{Q} / \mathbb{Z}=\bigcup_{n}\left(\frac{1}{n} \mathbb{Z} / \mathbb{Z}\right)^{*} \simeq \bigcup_{n} \operatorname{Gal}\left(\mathbb{Q}\left(\zeta_{n}\right) / \mathbb{Q}\right)
$$

and

$$
\bar{T} \simeq \bigcup_{\mathfrak{n}} \operatorname{Gal}\left(K_{\mathfrak{n}} / k\right)
$$

Thus one may think of $\bar{T}$ as an analogue of $\mathbb{Q} / \mathbb{Z}$.

An ordinary distribution on $k$ with values in an abelian group $V$ is a function $\Theta: \bar{T} \rightarrow V$ such that

$$
\Theta(a)=\sum_{\mathfrak{n} b=a} \Theta(b)
$$

for any integral ideal $\mathfrak{n}$ of $\mathbb{A}$ and $a \in \bar{T}$. Let $\mathcal{A}$ be the free abelian group generated by the symbols $[a]$ for $a \in \bar{T}$, and $\mathcal{A}_{\mathfrak{m}}$ the subgroup of $\mathcal{A}$ generated by the symbols $[a]$ for $a \in \bar{T}_{\mathfrak{m}}$. Then $G_{\mathfrak{m}}$ acts on $\mathcal{A}_{\mathfrak{m}}$ by $\sigma[a]=[\sigma a]$, and hence $G$ acts on $\mathcal{A}$.

Let $\mathcal{U}$ be the subgroup of $\mathcal{A}$ generated by the elements of the form

$$
E(\mathfrak{n}, a)=[a]-\sum_{\mathfrak{n} b=a}[b] \quad \text { for } \mathfrak{n} \in T_{\mathfrak{e}}, a, b \in \bar{T}^{*},
$$

and let $\mathcal{U}_{\mathfrak{m}}=\mathcal{U} \cap \mathcal{A}_{\mathfrak{m}}$. Note that, for $a \in \bar{T}_{\mathfrak{d}}^{\prime}$ and a prime ideal $\mathfrak{p}$, we have

$$
E(\mathfrak{p}, a)= \begin{cases}{[a]-\mathrm{Fr}_{\mathfrak{p}}^{-1}[a]-\sum_{\sigma \in G\left(K_{\mathfrak{d} p} / K_{\mathfrak{l}}\right)} \sigma\left[\mathfrak{p}^{-1} a\right]} & \text { if } \mathfrak{p} \nmid \mathfrak{d}, \\ {[a]-\sum_{\sigma \in G\left(K_{\mathfrak{d} \mathfrak{p}} / K_{\mathfrak{l}}\right)} \sigma\left[\mathfrak{p}^{-1} a\right]} & \text { if } \mathfrak{p} \mid \mathfrak{d} .\end{cases}
$$

Here $\operatorname{Fr}_{\mathfrak{p}}$ is the Frobenius map at $\mathfrak{p}$ in $G\left(K_{\mathfrak{d}} / k\right)$ and $\mathfrak{p}^{-1} a=\widetilde{\sigma}_{a}\left\{\mathfrak{p}^{-1} \mathfrak{d}_{a}^{-1}\right\}$, where $\widetilde{\sigma}_{a}$ is any extension of $\sigma_{a}$ to $K_{\mathfrak{p d}_{a}}$. Let $\mathbb{U}=\mathcal{A} / \mathcal{U}$ and $\mathbb{U}_{\mathfrak{m}}=\mathcal{A}_{\mathfrak{m}} / \mathcal{U}_{\mathfrak{m}}$. Then an ordinary distribution on $k$ with values in $V$ is a homomorphism from $\mathcal{A}$ to $V$ whose kernel contains $\mathcal{U}$, that is, a homomorphism from $\mathbb{U}$ to $V$. Define $u: \bar{T} \rightarrow \mathbb{U}$ to be the map induced by $a \mapsto[a]: \bar{T} \rightarrow \mathcal{A}$. Then $u$ 
is the universal ordinary distribution, i.e. the initial object in the category of ordinary distributions on $k$. By abuse of language we call $\mathbb{U}$ and $\mathbb{U}_{\mathfrak{m}}$ the universal ordinary distribution and the universal ordinary distribution of level $\mathfrak{m}$, respectively. It is known that the group $\mathbb{U}_{\mathfrak{m}}$ is free abelian of rank $\left|G_{\mathfrak{m}}\right|$ and $\mathbb{U}$ is free abelian $([\mathrm{A}],[\mathrm{Y}])$.

1.2. Anderson's resolution. In this section we briefly recall the construction of Anderson's resolutions of $\mathbb{U}$ and $\mathbb{U}_{\mathfrak{m}}$. Fix a total ordering " $<$ " in the set of prime ideals of $\mathbb{A}$. Let $\mathbb{L}$ be the free abelian group with basis $\{[a, \mathfrak{g}]\}$ indexed by $a \in \bar{T}$ and $\mathfrak{g} \in T_{\mathfrak{e}}$ square-free. We let $G$ act on $\mathbb{L}$ by $\sigma[a, \mathfrak{g}]:=[\sigma a, \mathfrak{g}]$. The degree of $[a, \mathfrak{g}]$ is defined to be $-o(\mathfrak{g})=-n(\mathfrak{g})$. Define, for a square-free integral ideal $\mathfrak{g}$ and a prime ideal $\mathfrak{p}$,

$$
\omega(\mathfrak{p}, \mathfrak{g})= \begin{cases}(-1)^{|\{\mathfrak{q}: \mathfrak{q} \mid \mathfrak{g}, \mathfrak{q}<\mathfrak{p}\}|} & \text { if } \mathfrak{p} \mid \mathfrak{g}, \\ 0 & \text { otherwise }\end{cases}
$$

and a differential operator $d$ by

$$
d[a, \mathfrak{g}]=\sum_{\mathfrak{p}} \omega(\mathfrak{p}, \mathfrak{g})\left(\left[a, \mathfrak{g} \mathfrak{p}^{-1}\right]-\sum_{\mathfrak{p} b=a}\left[b, \mathfrak{g} \mathfrak{p}^{-1}\right]\right) .
$$

Then $(\mathbb{L}, d)$ becomes a complex and the map $[a, \mathfrak{e}] \mapsto[a]$ induces an isomorphism $H^{0}(\mathbb{L}, d) \simeq \mathbb{U}$. For an integral ideal $\mathfrak{m}$, let $\mathbb{L}_{\mathfrak{m}}$ be the subgroup spanned by the symbols of the form $[a, \mathfrak{g}]$, where $\mathfrak{g} \mid \mathfrak{m}$ and $a \in \bar{T}_{\mathfrak{m g}^{-1}}$. Then $\mathbb{L}_{\mathfrak{m}}$ is $d$-stable and the map $[a, \mathfrak{e}] \mapsto[a]$ induces an isomorphism $H^{0}\left(\mathbb{L}_{\mathfrak{m}}, d\right) \simeq \mathbb{U}_{\mathfrak{m}}$. Let $M$ be a positive integer dividing $q^{\text {deg } \mathfrak{p}}-1$ for any $\mathfrak{p} \mid \mathfrak{m}$ and let $\mathbb{L}_{\mathfrak{m}, M}=\mathbb{L}_{\mathfrak{m}} / M \mathbb{L}_{\mathfrak{m}}$.

Following Anderson ([O, Proposition 3.2, 3.6 and Appendix by Anderson]), we have

THEOREM 1.2.1.

(a) For $\mathfrak{m} \in T_{\mathfrak{e}}$ the complex $\left(\mathbb{L}_{\mathfrak{m}}, d\right)$ is acyclic in nonzero degrees and $H^{0}\left(\mathbb{L}_{\mathfrak{m}}, d\right)=\mathbb{U}_{\mathfrak{m}}$.

(b) The complex $(\mathbb{L}, d)$ is acyclic in nonzero degrees.

(c) $H^{n}\left(\mathbb{L}_{\mathfrak{m}, M}\right)= \begin{cases}\mathbb{U}_{\mathfrak{m}} / M \mathbb{U}_{\mathfrak{m}} & \text { if } n=0, \\ 0 & \text { if } n \neq 0\end{cases}$

We give $\mathbb{L}_{\mathfrak{m}}$ a double complex structure. An element $[a, \mathfrak{g}]$ of $\mathbb{L}_{\mathfrak{m}}$ is of bidegree $\left(p_{1}, p_{2}\right)$ if $p_{1}=n\left(\mathfrak{d}_{a}\right)-n(\mathfrak{m})$ and $p_{2}=n(\mathfrak{m})-n\left(\mathfrak{d}_{a}\right)-n(\mathfrak{g})$. The differentials $d_{1}$ of bidegree $(1,0)$ and $d_{2}$ of bidegree $(0,1)$ are defined by

$$
d_{1}: L_{\mathfrak{m}}^{p_{1}, p_{2}} \rightarrow L_{\mathfrak{m}}^{p_{1}+1, p_{2}}, \quad[a, \mathfrak{g}] \mapsto-\sum_{\mathfrak{p}} \omega(\mathfrak{p}, \mathfrak{g}) N_{\mathfrak{p}}\left[\mathfrak{p}^{-1} a, \mathfrak{g} \mathfrak{p}^{-1}\right]
$$

and

$$
d_{2}: L_{\mathfrak{m}}^{p_{1}, p_{2}} \rightarrow L_{\mathfrak{m}}^{p_{1}, p_{2}+1}, \quad[a, \mathfrak{g}] \mapsto \sum_{\mathfrak{p}} \omega(\mathfrak{p}, \mathfrak{g})\left(1-\mathrm{Fr}_{\mathfrak{p}}\right)\left[a, \mathfrak{g} \mathfrak{p}^{-1}\right]
$$


Then $d=d_{1}+d_{2}, d_{1}^{2}=d_{2}^{2}=d_{1} d_{2}+d_{2} d_{1}=0$, and

$$
L_{\mathfrak{m}}^{p}=\bigoplus_{p_{1}+p_{2}=p} L_{\mathfrak{m}}^{p_{1}, p_{2}}
$$

2. Group cohomology of the universal ordinary distribution. In this section we fix a square-free integral ideal $\mathfrak{m}$. We use the group $H_{\mathfrak{m}}$ instead of $G_{\mathfrak{m}}$. The group cohomology for $G_{\mathfrak{m}}$ is very hard to understand because $G_{\mathfrak{m}}$ is not a direct product of inertia groups. Still the results we get here are weaker than those of $[\mathrm{O}]$ because our $\mathbb{A}$ is not a principal ideal domain. But when studying Euler systems in $\S 3$ we only consider principal ideals.

2.1. Double complex $\mathbb{K}$. Let $\mathfrak{m}^{\infty}$ be the formal $\infty$ th power of $\mathfrak{m}$. Let $\mathbb{K}$ be the free abelian group generated by the symbols $[a, \mathfrak{g}, \mathfrak{h}]$ indexed by $[a, \mathfrak{g}] \in \mathbb{L}_{\mathfrak{m}}$ and $\mathfrak{h} \mid \mathfrak{m}^{\infty}$. We say that $[a, \mathfrak{g}, \mathfrak{h}]$ has bidegree $(p, q)$ if $n(\mathfrak{g})=$ $o(\mathfrak{g})=-p$ and $o(\mathfrak{h})=q$. Let $d$ and $\delta$ be the differentials on $\mathbb{K}$ of bidegree $(1,0)$ and $(0,1)$, respectively given by

$$
d=\sum_{\mathfrak{p}} d_{\mathfrak{p}} \quad \text { and } \quad \delta=\sum_{\mathfrak{p}} \delta_{\mathfrak{p}},
$$

where

$$
\begin{aligned}
& d_{\mathfrak{p}}[a, \mathfrak{g}, \mathfrak{h}]=\omega(\mathfrak{p}, \mathfrak{g})\left(\left[a, \mathfrak{g p}^{-1}, \mathfrak{h}\right]-\sum_{\mathfrak{p} b=a}\left[b, \mathfrak{g} \mathfrak{p}^{-1}, \mathfrak{h}\right]\right), \\
& \delta_{\mathfrak{p}}[a, \mathfrak{g}, \mathfrak{h}]=(-1)^{o(\mathfrak{g})+\sum_{\mathfrak{q}<\mathfrak{p}} \operatorname{ord}_{\mathfrak{q}} \mathfrak{h}} \begin{cases}\left(1-\sigma_{\mathfrak{p}}\right)[a, \mathfrak{g}, \mathfrak{h} \mathfrak{p}] & \text { if } \operatorname{ord}_{\mathfrak{p}} \mathfrak{h} \text { is even, } \\
N_{\mathfrak{p}}[a, \mathfrak{g}, \mathfrak{h p}] & \text { otherwise. }\end{cases}
\end{aligned}
$$

Note that $d$ is induced by the differential $d$ on $\mathbb{L}$, and thus we can decompose $d$ into $d_{1}+d_{2}$. Let $\mathbb{K}_{M}=\mathbb{K} \otimes \mathbb{Z} / M$. We usually write $\mathbb{M}^{*}$ or $\mathbb{M}^{*, *}$ for a module $\mathbb{M}$ to specify the complex $(\mathbb{M}, d)$ or the double complex $(\mathbb{M}, d, \delta)$.

Set

$$
\mathbf{U}^{*}:=\frac{\left\langle[a, \mathfrak{h}]: a \in \bar{T}_{\mathfrak{m}}, \mathfrak{h} \mid \mathfrak{m}^{\infty}\right\rangle}{\left\langle[a, \mathfrak{h}]-\sum_{\mathfrak{p} b=a}[b, \mathfrak{h}]: a \in \bar{T}_{\mathfrak{m p}^{-1}}, \mathfrak{h} \mid \mathfrak{m}^{\infty}\right\rangle} .
$$

We consider $\mathbf{U}^{*}$ as the double complex $\left(\mathbf{U}^{*, *} ; 0, \delta\right)$ concentrated on the vertical axis. Let $\mathbf{u}: \mathbb{K}^{*, *} \rightarrow \mathbf{U}^{*, *}$ be the map

$$
[a, \mathfrak{g}, \mathfrak{h}] \mapsto \begin{cases}{[a, \mathfrak{h}]} & \text { if } \mathfrak{g}=\mathfrak{e}, \\ 0 & \text { otherwise. }\end{cases}
$$

Proposition 2.1.1 ([O, Proposition 5.1]). The map $\mathbf{u}$ is a quasi-isomorphism between $\mathbb{K}^{*, *}$ and $\mathbf{U}^{*, *}$. Therefore

$$
H_{\text {total }}^{*}\left(\mathbb{K}^{*, *}\right)=H^{*}\left(H_{\mathfrak{m}}, \mathbb{U}_{\mathfrak{m}}\right) \quad \text { and } \quad H_{\text {total }}^{*}\left(\mathbb{K}_{M}^{*, *}\right)=H^{*}\left(H_{\mathfrak{m}}, \mathbb{U}_{\mathfrak{m}} / M \mathbb{U}_{\mathfrak{m}}\right) \text {. }
$$


2.2. Spectral sequence for $\mathbb{K}^{*, *}$. Now we study the spectral sequence of $\mathbb{K}^{*, *}$ from the first filtration. Note first that

$$
E_{1}^{p, q}\left(\mathbb{K}^{*, *}\right)=H_{\delta}^{q}\left(\mathbb{K}^{p, *}\right)=H^{q}\left(H_{\mathfrak{m}}, L^{p}\right) .
$$

Since $L^{p}=\bigoplus_{p_{1}+p_{2}=p} L^{p_{1}, p_{2}}=\bigoplus_{o(\mathfrak{g})=-p} \bigoplus_{\mathfrak{g}\left|\mathfrak{g}^{\prime}\right| \mathfrak{m}} L_{\mathfrak{g}^{\prime}, \mathfrak{g}}$, where $L_{\mathfrak{g}^{\prime}, \mathfrak{g}}:=\langle[a, \mathfrak{g}]$ : $\left.a \in \bar{T}_{\mathfrak{m g}^{\prime}-1}\right\rangle$, we have

$$
E_{1}^{p, q}\left(\mathbb{K}^{*, *}\right)=\bigoplus_{o(\mathfrak{g})=-p} \bigoplus_{\mathfrak{g}\left|\mathfrak{g}^{\prime}\right| \mathfrak{m}} H^{q}\left(H_{\mathfrak{m}}, L_{\mathfrak{g}^{\prime}, \mathfrak{g}}\right)
$$

$H^{q}\left(H_{\mathfrak{m}}, L_{\mathfrak{g}^{\prime}, \mathfrak{g}}\right)$ is isomorphic to $H^{q}\left(H_{\mathfrak{g}^{\prime}}, \mathbb{Z}\left[G_{\mathfrak{e}}\right]\right)$ by Shapiro's lemma. We write this as $H^{q}\left(H_{\mathfrak{g}^{\prime}, \mathfrak{g}}, \mathbb{Z}\left[G_{\mathfrak{e}}\right]\right)$ to keep track of $\mathfrak{g}$.

The induced differential $\bar{d}_{1}$ is exactly the map

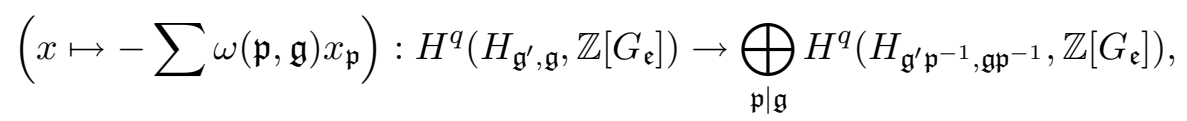

where $x_{\mathfrak{p}}$ is the restriction of $x$ in $H^{q}\left(H_{\mathfrak{g}^{\prime} \mathfrak{p}^{-1}, \mathfrak{g p}} \mathfrak{p}^{-1}, \mathbb{Z}\left[G_{\mathfrak{e}}\right]\right)$. The induced differential $\bar{d}_{2}$ on $E_{1}$ is

$$
\bar{d}_{2}(\tau)=\sum_{\mathfrak{p} \mid \mathfrak{g}} \omega(\mathfrak{p}, \mathfrak{g})\left(1-\operatorname{Fr}_{\mathfrak{p}}^{-1}\right) \tau
$$

after identifying $H^{q}\left(H_{\mathfrak{g}^{\prime}, \mathfrak{g}}, \mathbb{Z}\left[G_{\mathfrak{e}}\right]\right)=H^{q}\left(H_{\mathfrak{g}^{\prime}, \mathfrak{g} \mathfrak{p}^{-1}}, \mathbb{Z}\left[G_{\mathfrak{e}}\right]\right)$.

Unlike the rational case, $\bar{d}_{2}$ may not equal to 0 because of the nontriviality of the class group of $k$. But if $\mathfrak{m}$ is divisible only by principal prime ideals, then $\bar{d}_{2}=0$.

2.3. The complex $\mathbb{Q}^{*, *}$. Put $\mathbb{S}^{*, *}=\left\langle[a, \mathfrak{g}, \mathfrak{h}] \in \mathbb{K}^{*, *}: a \notin \bar{T}_{\mathfrak{e}}\right.$ if $\left.\mathfrak{g} \mid \mathfrak{h}\right\rangle$, and

$$
\mathbb{Q}^{*, *}=\mathbb{K}^{*, *} / \mathbb{S}^{*, *}=\left\langle[a, \mathfrak{g}, \mathfrak{h}]: a \in \bar{T}_{\mathfrak{e}}, \mathfrak{g} \mid \mathfrak{h}\right\rangle .
$$

The induced differential $d$ on $\mathbb{Q}^{*, *}$ is

$$
d[a, \mathfrak{g}, \mathfrak{h}]=\sum_{\mathfrak{p}} \omega(\mathfrak{p}, \mathfrak{g})\left(1-\operatorname{Fr}_{\mathfrak{p}}^{-1}\right)\left[a, \mathfrak{g p}^{-1}, \mathfrak{h}\right]
$$

which is not 0 , in general.

Proposition 2.3.1. The quotient map $f: \mathbb{K}^{*, *} \rightarrow \mathbb{Q}^{*, *}$ is a quasiisomorphism.

Proof. Let $E_{1}\left(\mathbb{K}^{*, *}\right)=\left(E_{1}^{p, q}(\mathbb{K})\right)$. Then as in $[\mathrm{O}, \S 5.3]$, for each $q, E_{1}^{*, q}$ is the double complex $\mathcal{M}_{q}=\left(M_{q}^{p_{1}, p_{2}}, \bar{d}_{1}, \bar{d}_{2}\right)$, where

$$
M_{q}^{p_{1}, p_{2}}=\bigoplus_{\substack{\mathfrak{g} \mid \mathfrak{g}^{\prime} \\ o\left(\mathfrak{g}^{\prime}\right)=-p_{1} \\ o(\mathfrak{g})=-p_{1}-p_{2}}} H^{q}\left(H_{\mathfrak{g}^{\prime}, \mathfrak{g}}, \mathbb{Z}\right)\left[G_{\mathfrak{e}}\right],
$$


with $\bar{d}_{1}$ and $\bar{d}_{2}$ as in $\S 2.2$. Let

$$
\begin{aligned}
& \mathcal{L}_{\mathfrak{g}}^{\prime *}:=\left\langle[a, \mathfrak{g}, \mathfrak{h}]: a \in \bar{T}_{\mathfrak{e}}, \mathfrak{g} \mid \mathfrak{h}, *=o(\mathfrak{h})\right\rangle, \\
& L_{\mathfrak{g}}^{\prime *}:=\langle[\{\mathbb{A}\}, \mathfrak{g}, \mathfrak{h}]: \mathfrak{g} \mid \mathfrak{h}, *=o(\mathfrak{h})\rangle .
\end{aligned}
$$

Then $\mathcal{L}_{\mathfrak{g}}^{\prime *}=L_{\mathfrak{g}}^{\prime *}\left[G_{\mathfrak{e}}\right]$. Since $L_{\mathfrak{g}}^{\prime *}$ is $\bar{d}_{1}$-stable, one can follow the proof of Proposition 5.4 of $[\mathrm{O}]$. The $E_{1}$-term of the spectral sequence for the second filtration of the double complex $\mathcal{M}_{q}$ is given, for $m=n(\mathfrak{m})$, by

$$
E_{1}^{p_{1}, p_{2}}\left(\mathcal{M}_{q}\right)= \begin{cases}\bigoplus_{o(\mathfrak{g})=m-p_{2}} H^{q}\left(\mathcal{L}_{\mathfrak{g}}^{\prime *}\right)=\bigoplus_{o(\mathfrak{g})=m-p_{2}} H^{q}\left(L_{\mathfrak{g}}^{\prime *}\right)\left[G_{\mathfrak{e}}\right] & \text { if } p_{1}=-m, \\ 0 & \text { otherwise. }\end{cases}
$$

Therefore $\mathcal{M}_{q}$ degenerates at $E_{2}$ and

$$
E_{2}^{p, q}\left(\mathbb{K}^{*, *}\right)=H^{m+p}\left(\mathcal{N}_{q}\right)
$$

where

$$
\mathcal{N}_{q}=\left(N_{p_{2}}=\bigoplus_{o(\mathfrak{g})=m-p_{2}} H^{q}\left(L_{\mathfrak{g})}^{\prime}\left[G_{\mathfrak{e}}\right], d_{2}\right) .\right.
$$

On the other hand, $\mathbb{Q}^{*, *}=\bigoplus_{\mathfrak{g} \mid \mathfrak{m}} \mathcal{L}_{\mathfrak{g}}^{\prime *}$. Since $d_{1}=0$ on $\mathbb{Q}^{*, *}$, we have $E_{1}^{p, q}\left(\mathbb{Q}^{*, *}\right)=\bigoplus_{o(\mathfrak{g})=-p} H^{q}\left(L_{\mathfrak{g}}^{\prime}\right)\left[G_{\mathfrak{e}}\right]$, and so $E_{2}^{p, q}\left(\mathbb{Q}^{*, *}\right)=H^{m+p}\left(\mathcal{N}_{q}\right)$. Therefore $f_{2}$ is an isomorphism, which implies that $f$ is a quasi-isomorphism.

The quotient map also induces a quasi-isomorphism between $\mathbb{K}_{M}^{*, *}$ and $\mathbb{Q}_{M}^{*, *}$. The differential $\delta$ on $\mathbb{Q}_{M}^{*, *}$ is 0 . But since $d$ is not 0 , the spectral sequence for $\mathbb{Q}_{M}^{*, *}$ does not degenerate at $E_{1}$. Thus it is not easy to give a basis of $H^{*}\left(H_{\mathfrak{m}}, \mathbb{U}_{\mathfrak{m}}\right)$ and $H^{*}\left(H_{\mathfrak{m}}, \mathbb{U}_{\mathfrak{m}} / M \mathbb{U}_{\mathfrak{m}}\right)$. However we can get an explicit basis for $H^{0}\left(H_{\mathfrak{m}}, \mathbb{U}_{\mathfrak{m}} / M \mathbb{U}_{\mathfrak{m}}\right)$.

TheOREM 2.3.2. There is a basis of $H^{0}\left(H_{\mathfrak{m}}, \mathbb{U}_{\mathfrak{m}} / M \mathbb{U}_{\mathfrak{m}}\right)$ consisting of

$$
\left\{c_{\mathfrak{g}, \sigma} \in H^{0}\left(H_{\mathfrak{m}}, \mathbb{U}_{\mathfrak{m}} / M \mathbb{U}_{\mathfrak{m}}\right): \mathfrak{g} \mid \mathfrak{m}, \sigma \text { a representative of } G_{\mathfrak{e}} / A_{\mathfrak{g}}\right\}
$$

where $A_{\mathfrak{g}}$ is the subgroup of $G_{\mathfrak{e}}$ generated by $\operatorname{Fr}_{\mathfrak{p}}$ with $\mathfrak{p} \mid \mathfrak{g}$.

Proof. Write $x(\mathfrak{g}, \mathfrak{h})$ to denote $[\{\mathfrak{e}\}, \mathfrak{g}, \mathfrak{h}]$. For $\mathfrak{g}|\mathfrak{m}, \mathfrak{h}| \mathfrak{m}^{\infty}$ and $\mathfrak{g} \mid \mathfrak{h}$, let $\mathbf{C}(\mathfrak{g}, \mathfrak{h})$ be the complex

$$
\begin{aligned}
\quad \cdots 0 \rightarrow \mathbb{Z} / M\left[G_{\mathfrak{e}}\right] x(\mathfrak{g}, \mathfrak{h}) \stackrel{d}{\rightarrow} \bigoplus_{\mathfrak{p} \mid \mathfrak{g}} \mathbb{Z} / M\left[G_{\mathfrak{e}}\right] x\left(\mathfrak{g p} \mathfrak{p}^{-1}, \mathfrak{h}\right) \rightarrow \\
\quad \cdots \rightarrow \mathbb{Z} / M\left[G_{\mathfrak{e}}\right] x(\mathfrak{e}, \mathfrak{h}) \rightarrow 0,
\end{aligned}
$$

where $d x\left(\mathfrak{g}^{\prime}, \mathfrak{h}\right)=\sum_{\mathfrak{p} \mid \mathfrak{g}^{\prime}} w\left(\mathfrak{p}, \mathfrak{g}^{\prime}\right)\left(1-\operatorname{Fr}_{\mathfrak{p}}\right) x\left(\mathfrak{g}^{\prime} \mathfrak{p}^{-1}, \mathfrak{h}\right)$. Then the kernel of $d$ at degree 0 is $\mathbb{Z} / M\left[G_{\mathfrak{e}}\right] s\left(A_{\mathfrak{g}}\right) x(\mathfrak{g}, \mathfrak{g})$ if $\mathfrak{g}=\mathfrak{h}$, and 0 otherwise. Therefore the set

$$
\left\{\sigma s\left(A_{\mathfrak{g}}\right) x(\mathfrak{g}, \mathfrak{g}): \sigma \text { a representative of } G_{\mathfrak{e}} / A_{\mathfrak{g}}\right\}
$$

is a basis of the 0 th cohomology of the complex $\mathbf{C}(\mathfrak{g}, \mathfrak{g})$. It is easy to see that $\mathbb{Q}_{M}^{*, *}$ is the direct sum of the complexes $\mathbf{C}(\mathfrak{g}, \mathfrak{h})$ for $\mathfrak{g}|\mathfrak{m}, \mathfrak{h}| \mathfrak{m}^{\infty}$ and $\mathfrak{g} \mid \mathfrak{h}$. Let 
$C(\mathfrak{g}, \sigma)$ be the cocycle of $\mathbb{K}_{M}^{*, *}$ lifting $\sigma s\left(A_{\mathfrak{g}}\right) x(\mathfrak{g}, \mathfrak{g})$, and $c_{\mathfrak{g}, \sigma}$ be the image of $\mathbf{u}(C(\mathfrak{g}, \sigma))$ in $H^{0}\left(H_{\mathfrak{m}}, \mathbb{U}_{\mathfrak{m}} / M \mathbb{U}_{\mathfrak{m}}\right)$. Hence we get the result.

The basis of $H^{0}\left(H_{\mathfrak{m}}, \mathbb{U}_{\mathfrak{m}} / M \mathbb{U}_{\mathfrak{m}}\right)$ constructed in the proof of Theorem 2.3.2 will be called the canonical basis.

If $\mathfrak{m}$ is divisible only by principal prime ideals, then the differential $d$ on $\mathbb{Q}_{M}^{*, *}$ is trivial, and it is easy to compute the cohomology of the complex $\mathbb{K}_{M}^{*, *}$. In this case we actually have

$$
\mathbb{U}_{\mathfrak{m}}=\bigoplus_{\sigma \in G_{\mathfrak{e}}} \mathbb{U}_{\mathfrak{m}, \sigma}
$$

and most of the results in $[\mathrm{O}]$ would hold for $\mathbb{U}_{\mathfrak{m}, \sigma}$ for any $\sigma \in G_{\mathfrak{e}}$. In particular, we have

Corollary 2.3.3. For $\mathfrak{g} \mid \mathfrak{m}$ and $\sigma \in G_{\mathfrak{e}}$, let $c_{\mathfrak{g}, \sigma}$ be the image in $H^{0}\left(H_{\mathfrak{m}}, \mathbb{U}_{\mathfrak{m}, \sigma} / M \mathbb{U}_{\mathfrak{m}, \sigma}\right)$ of the cocycle lifting $\sigma x(\mathfrak{g}, \mathfrak{g})$. Then $\left\{c_{\mathfrak{g}, \sigma}\right\}$ is the canonical basis of $H^{0}\left(H_{\mathfrak{m}}, \mathbb{U}_{\mathfrak{m}, \sigma} / M \mathbb{U}_{\mathfrak{m}, \sigma}\right)$.

Define the diagonal shift operator $\Delta_{\mathfrak{l}}$ on $\mathbb{K}$ by

$$
\Delta_{\mathfrak{l}}[a, \mathfrak{g}, \mathfrak{h}]:= \begin{cases}{\left[a, \mathfrak{g l}^{-1}, \mathfrak{h l}^{-1}\right]} & \text { if } \mathfrak{l} \mid \mathfrak{g} \text { and } \mathfrak{l} \mid \mathfrak{h}, \\ 0 & \text { otherwise. }\end{cases}
$$

Then $\Delta_{\mathfrak{l}}$ induces endomorphisms of $H^{0}(G, \mathbb{U} / M \mathbb{U})$ and of $H^{0}\left(G, \mathbb{U}_{\mathfrak{m}} / M \mathbb{U}_{\mathfrak{m}}\right)$.

3. Euler systems and Kolyvagin classes. Let $F$ be a finite abelian extension of $k$, containing the Hilbert class field $K_{\mathfrak{e}}$, such that $\infty$ splits completely. Let $M$ be a positive integer prime to $q(q-1)$. Let $\mathbf{m}$ be the formal product of prime ideals $\mathfrak{p}$, which splits completely in $F$ and $M \mid q^{\operatorname{deg} \mathfrak{p}}-1$.

Let $F_{\mathfrak{m}}=F K_{\mathfrak{m}}$ and $\mathcal{O}_{\mathfrak{m}}$ be the integral closure of $\mathbb{A}$ in $F_{\mathfrak{m}}$. Let $K_{\mathfrak{m}}$ be the compositum of all fields $K_{\mathfrak{m}}$ with $\mathfrak{m} \mid \mathbf{m}$, and $F_{\mathbf{m}}=F K_{\mathbf{m}}$. Then $G^{\prime}=\operatorname{Gal}\left(F_{\mathbf{m}} / F\right)$ is isomorphic to $H_{\mathfrak{m}}=\lim _{\mathfrak{m} \mid \mathbf{m}} H_{\mathfrak{m}}$. Put $\mathcal{O}:=\mathcal{O}_{\mathfrak{e}}$ and $\mathcal{O}_{\mathfrak{m}}:=\bigcup_{\mathfrak{m} \mid \mathbf{m}} \mathcal{O}_{\mathfrak{m}}$. For each integral ideal $\mathfrak{m}$ dividing $\mathbf{m}$ and a prime ideal $\mathfrak{l}$, let $\mathcal{O}_{\mathfrak{m},(\mathfrak{l})}$ be the localization of $\mathcal{O}_{\mathfrak{m}}$ with respect to the multiplicative system of elements prime to $\mathfrak{l}, \mathcal{O}_{(\mathfrak{l})}:=\mathcal{O}_{\mathfrak{e},(\mathfrak{l})}$, and $\mathcal{O}_{\mathbf{m},(\mathfrak{l})}:=\bigcup_{\mathfrak{m} \mid \mathbf{m}} \mathcal{O}_{\mathfrak{m},(\mathfrak{l})}$. Let $\mathbb{U}_{\mathbf{m}}=\bigcup_{\mathfrak{m} \mid \mathbf{m}} \mathbb{U}_{\mathfrak{m}}$ and $\bar{T}_{\mathbf{m}}=\bigcup_{\mathfrak{m} \mid \mathbf{m}} \bar{T}_{\mathfrak{m}}$.

3.1. The universal Euler system and the universal Kolyvagin class. Let $\bar{T}_{\mathbf{m}}^{1}$ be the subset of $\bar{T}_{\mathbf{m}}$ consisting of elements $a$ such that the restriction of $\sigma_{a}$ is the identity on $K_{\mathfrak{e}}$. Let $\mathbb{U}_{\mathbf{m}}^{1}=\mathbb{U}_{\mathbf{m} \text {,id. }}$. Let $\mathfrak{l} \mid \mathfrak{m}$ be a prime ideal. For $a, b \in \bar{T}_{\mathbf{m}}^{1}$, we write $a \sim_{\mathfrak{l}} b$ if one of the following two conditions is satisfied:

1) $\mathfrak{l} \mid \mathfrak{d}_{a}=\mathfrak{d}_{b}$ and $\sigma_{a}=\sigma_{b}$ on $K_{\mathfrak{d}_{a} \mathfrak{l}^{-1}}$

2) $\mathfrak{d}_{a}=\mathfrak{d}_{b} \mathfrak{l}$ and $\sigma_{a}=\sigma_{b} \operatorname{Fr}_{\mathfrak{l}}$ on $K_{\mathfrak{d}_{b}}$.

Let $I_{\mathfrak{l}}$ be the subgroup generated by the elements of $\mathbb{U}_{\mathbf{m}}^{1}$ of the form $[a]-[b]$ 
with $a \sim_{\mathfrak{l}} b$. It is easy to see that $I_{\mathfrak{l}}$ is $H_{\mathbf{m}}$-stable and

$$
\left(\sigma_{\mathfrak{l}}-1\right) \mathbb{U}_{\mathbf{m}}^{1} \subset I_{\mathfrak{l}} .
$$

Thus we may view $\mathbb{U}_{\mathbf{m}}^{1} / I_{\mathfrak{l}}$ as an $H_{\mathbf{m}} / H_{\mathfrak{l}}$-module, and we have an exact sequence

$$
0 \rightarrow \mathbb{U}_{\mathbf{m}^{-1}}^{1} \stackrel{|\mathfrak{l}|-\mathrm{Fr}_{\mathfrak{l}}}{\longrightarrow} \mathbb{U}_{\mathbf{m l}^{-1}}^{1} \rightarrow \mathbb{U}_{\mathbf{m}}^{1} / I_{\mathfrak{l}} \rightarrow 0
$$

where the map $\mathbb{U}_{\mathbf{m} \mathfrak{l}^{-1}}^{1} \rightarrow \mathbb{U}_{\mathbf{m}}^{1} / I_{\mathfrak{l}}$ is induced by the inclusion and $|\mathfrak{l}|=q^{\operatorname{deg} \mathfrak{l}}$. As in the rational number field case we have the following analogue of Proposition 3.7 of $[\mathrm{AO}]$.

Proposition 3.1.1 (cf. [AO, Proposition 3.7]). For every prime ideal $\mathfrak{l}$ dividing $\mathbf{m}$ there exists a unique homomorphism

$$
D_{\mathfrak{l}}: H^{0}\left(H_{\mathbf{m}}, \mathbb{U}_{\mathbf{m}}^{1} / M \mathbb{U}_{\mathbf{m}}^{1}\right) \rightarrow H^{0}\left(H_{\mathbf{m}}, \mathbb{U}_{\mathbf{m} \mathfrak{l}^{-1}}^{1} / M \mathbb{U}_{\mathbf{m} \mathfrak{l}^{-1}}^{1}\right)
$$

such that

$$
\frac{\left(\sigma_{\mathfrak{l}}-1\right) x}{M} \equiv \frac{\left(|\mathfrak{l}|-\mathrm{Fr}_{\mathfrak{l}}\right) y}{M} \bmod I_{\mathfrak{l}} \Leftrightarrow D_{\mathfrak{l}}\left(x \bmod M \mathbb{U}_{\mathbf{m}}^{1}\right)=y \bmod M \mathbb{U}_{\mathbf{m l}^{-1}}^{1}
$$

for all $x \in \mathbb{U}_{\mathbf{m}}^{1}$ representing a class in $H^{0}\left(H_{\mathbf{m}}, \mathbb{U}_{\mathbf{m}}^{1} / M \mathbb{U}_{\mathbf{m}}^{1}\right)$ and $y \in \mathbb{U}_{\mathbf{m} \mathfrak{l}^{-1}}^{1}$ representing a class in $H^{0}\left(H_{\mathbf{m}}, \mathbb{U}_{\mathbf{m}^{-1}}^{1} / M \mathbb{U}_{\mathbf{m} \mathfrak{l}^{-1}}^{1}\right)$. Moreover,

$$
D_{\mathfrak{l}} H^{0}\left(H_{\mathbf{m}}, \mathbb{U}_{\mathfrak{m}}^{1} / M \mathbb{U}_{\mathfrak{m}}^{1}\right) \subset H^{0}\left(H_{\mathbf{m}}, \mathbb{U}_{\mathfrak{m} \mathfrak{l}^{-1}}^{1} / M \mathbb{U}_{\mathfrak{m} \mathfrak{l}^{-1}}^{1}\right)
$$

for all integral ideals $\mathfrak{m}$ dividing $\mathbf{m}$ and divisible by $\mathfrak{l}$.

The difference in the proof of Proposition 3.1.1 from the classical case is the definition of $\varrho_{\mathfrak{l}}$, which in our case is

$$
\varrho_{\mathfrak{r}}([a])=\operatorname{Fr}_{\mathfrak{l}}^{-1}[\mathfrak{l} \cdot a]
$$

For each $\mathfrak{m} \mid \mathbf{m}$ there exists a unique element $\eta_{\mathfrak{m}}$ in $H_{\mathfrak{m}}$ whose restriction to $K_{\mathfrak{p}}$ is $\prod_{\mathfrak{p} \neq \mathfrak{q} \mid \mathfrak{m}} \operatorname{Fr}_{\mathfrak{q}}$ for $\mathfrak{p} \mid \mathfrak{m}$, by the Chinese remainder theorem. Let $x_{\mathfrak{m}} \in \mathbb{U}_{\mathfrak{m}}$ be the class represented by $\eta_{\mathfrak{m}}\left\{\mathfrak{m}^{-1}\right\}$. Then from the relation (1.1.2) and the definition of $I_{\mathfrak{l}}$, we have

$$
N_{\mathfrak{l}} x_{\mathfrak{m}}=\left(\mathrm{Fr}_{\mathfrak{l}}-1\right) x_{\mathfrak{m l}^{-1}} \quad \text { and } \quad x_{\mathfrak{m}} \equiv x_{\mathfrak{m l}^{-1}} \bmod I_{\mathfrak{l}}
$$

for all integral ideals $\mathfrak{m}$ dividing $\mathbf{m}$ and prime ideals $\mathfrak{l}$ dividing $\mathfrak{m}$.

We call the family

$$
\left\{x_{\mathfrak{m}} \in \mathbb{U}_{\mathfrak{m}}^{1} \subset \mathbb{U}_{\mathbf{m}}^{1}\right\}_{\mathfrak{m} \mid \mathbf{m}}
$$

the universal Euler system.

For each prime ideal $\mathfrak{l} \mid \mathfrak{m}$, one sees easily that

$$
\left(\sigma_{\mathfrak{l}}-1\right) N_{\mathfrak{m}}^{\prime} x_{\mathfrak{m}} \equiv-N_{\mathfrak{l}} N_{\mathfrak{m l}^{-1}}^{\prime} x_{\mathfrak{m}} \equiv\left(\mathrm{Fr}_{\mathfrak{l}}-1\right) N_{\mathfrak{m l}^{-1}}^{\prime} x_{\mathfrak{m} \mathfrak{l}^{-1}} \equiv 0 \bmod M \mathbb{U}_{\mathfrak{m}}^{1} .
$$

Hence $N_{\mathfrak{m}}^{\prime} x_{\mathfrak{m}} \in \mathbb{U}_{\mathfrak{m}}^{1} \subset \mathbb{U}_{\mathfrak{m}}^{1}$ represents a class $c_{\mathfrak{m}} \in H^{0}\left(H_{\mathfrak{m}}, \mathbb{U}_{\mathfrak{m}}^{1} / M \mathbb{U}_{\mathfrak{m}}^{1}\right)$. We call $c_{\mathfrak{m}}$ the universal Kolyvagin class indexed by $\mathfrak{m}$. Again we have the following analogue of Proposition 4.5 of [AO]. 
Proposition 3.1.2 (cf. [AO, Proposition 4.5]). The family $\left\{c_{\mathfrak{m}}\right\}$ of the universal Kolyvagin classes satisfies

$$
\mathfrak{l} \mid \mathfrak{m} \Rightarrow D_{\mathfrak{l}} c_{\mathfrak{m}}=c_{\mathfrak{m l}^{-1}} .
$$

We call the relation $(*)$ the universal Kolyvagin recursion.

Remark. As in the classical case ([AO, Proposition 5.8]) one can show that the endomorphism of $H^{0}\left(H_{\mathfrak{m}}, \mathbb{U}_{\mathfrak{m}}^{1} / M \mathbb{U}_{\mathfrak{m}}^{1}\right)$ induced by the diagonal shift operator $\Delta_{\mathfrak{l}}$ coincides with $D_{\mathfrak{l}}$. Thus the canonical basis $\left\{c_{\mathfrak{m}, \text { id }}\right\}$ satisfies the universal Kolyvagin recursion and the universal Kolyvagin classes $\left\{c_{\mathfrak{m}}\right\}$ form a basis of $H^{0}\left(H_{\mathbf{m}}, \mathbb{U}_{\mathbf{m}}^{1} / M \mathbb{U}_{\mathbf{m}}^{1}\right)$.

3.2. Euler system and Kolyvagin class. We call a collection $\left\{\xi_{\mathfrak{m}} \in F_{\mathfrak{m}}^{*}\right\}_{\mathfrak{m} \mid \mathbf{m}}$ an Euler system if

$$
\begin{gathered}
\xi_{\mathfrak{m}} \in \mathcal{O}_{\mathfrak{m}}^{*} \quad \text { for } \mathfrak{m} \neq \mathfrak{e}, \\
\xi_{\mathfrak{m}}^{N_{\mathfrak{l}}}=\xi_{\mathfrak{m l}^{-1}}^{\mathrm{Fr}_{\mathfrak{l}}-1}
\end{gathered}
$$

and

$$
\xi_{\mathfrak{m}} \equiv \xi_{\mathfrak{m} \mathfrak{l}^{-1}} \quad \text { modulo the radical of the ideal } \mathfrak{r O}_{\mathfrak{m}},
$$

for a prime ideal $\mathfrak{l}$ dividing $\mathfrak{m}$. In $[\mathrm{AO}]$ it is assumed in the definition that $\xi_{\mathfrak{e}}$ is a unit, which is not necessary.

EXAMPLE 3.2.1. Let $F=K_{\mathfrak{r}}^{+}$, the maximal real subfield of $K_{\mathfrak{r}}$, and $F_{\mathfrak{m}}=F K_{\mathfrak{m}}$. Let $\mathfrak{q}$ be a prime ideal not dividing $\mathfrak{r}$ and $\lambda$ be a nonzero $\mathfrak{q}$ torsion point of a sign-normalized rank 1 Drinfeld module $\varrho$. For $\mathfrak{m} \mid \mathbf{m}$ define

$$
\xi_{\mathfrak{m}}=N_{K_{\mathfrak{m} \mathfrak{q} r} / F_{\mathfrak{m}}}\left(\lambda-\sum_{\mathfrak{p} \mid \mathfrak{m}} \lambda(\mathfrak{p})\right),
$$

where $\lambda(\mathfrak{p})$ is a fixed nonzero $\mathfrak{p}$ torsion point of $\varrho$. Then $\left\{\xi_{\mathfrak{m}}\right\}_{\mathfrak{m}}$ is an Euler system.

Example 3.2.2. Let $F$ and $F_{\mathfrak{m}}$ be as in Example 3.2.1. Define

$$
\xi_{\mathfrak{m}}=N_{K_{\mathfrak{m} \mathfrak{r}} / F_{\mathfrak{m}}}\left(\lambda-\sum_{\mathfrak{p} \mid \mathfrak{m}} \lambda(\mathfrak{p})\right),
$$

where $\lambda$ is a nonzero $\mathfrak{r}$ torsion point of $\varrho$. Then $\left\{\xi_{\mathfrak{m}}\right\}_{\mathfrak{m}}$ is an Euler system.

REMARK 3.2.3. Let $F(\mathfrak{p})$ be a subfield of $F K_{\mathfrak{p}}$ of degree $M$ over $F$ and $F(\mathfrak{m})=\prod_{\mathfrak{p} \mid \mathfrak{m}} F(\mathfrak{p})$, as in $[\mathrm{XZ}]$. Then the two Euler systems in the proof of Theorem 3.3 in [XZ] are just the norm $N_{F_{\mathfrak{m}} / F(\mathfrak{m})} \xi_{\mathfrak{m}}$ of $\xi_{\mathfrak{m}}$ in the examples above. It would be an interesting problem to find an example which does not arise in this way.

As in [AO] we can identify $H^{0}\left(H_{\mathfrak{m}}, F_{\mathfrak{m}}^{*} / F_{\mathfrak{m}}^{* M}\right)$ with $F^{*} / F^{* M}$. Moreover, (image of $H^{0}\left(H_{\mathfrak{m}}, \mathcal{O}_{\mathfrak{m}}^{*} / \mathcal{O}_{\mathfrak{m}}^{* M}\right)$ in $\left.H^{0}\left(H_{\mathfrak{m}}, F_{\mathfrak{m}}^{*} / F_{\mathfrak{m}}^{* M}\right)\right) \subset \mathcal{O}_{(\mathfrak{l})}^{*} / \mathcal{O}_{(\mathfrak{l})}^{* M}$ for all prime ideals $\mathfrak{l}$ not dividing $\mathfrak{m}$. 
Let $\left\{\xi_{\mathfrak{m}}\right\}$ be an Euler system. Fix an integral ideal $\mathfrak{m} \neq \mathfrak{e}$ dividing $\mathbf{m}$. For each prime ideal $\mathfrak{l}$ dividing $\mathfrak{m}$ one can show that $\xi_{\mathfrak{m}}^{N_{\mathfrak{m}}^{\prime}\left(1-\sigma_{\mathfrak{l}}\right)} \equiv 1 \bmod \mathcal{O}_{\mathfrak{m}}^{* M}$, and so

$$
\xi_{\mathfrak{m}}^{N_{\mathfrak{m}}^{\prime}} \bmod \mathcal{O}_{\mathfrak{m}}^{* M} \in H^{0}\left(H_{\mathfrak{m}}, \mathcal{O}_{\mathfrak{m}}^{*} / \mathcal{O}_{\mathfrak{m}}^{* M}\right)
$$

Therefore there exists a unique class $\kappa_{\mathfrak{m}} \in F^{*} / F^{* M}$ such that

$$
\xi_{\mathfrak{m}}^{N_{\mathfrak{m}}^{\prime}} \equiv \kappa_{\mathfrak{m}} \bmod F_{\mathfrak{m}}^{* M}
$$

and moreover, we have

$$
\mathfrak{m} \neq \mathfrak{e},(\mathfrak{l}, \mathfrak{m})=\mathfrak{e} \Rightarrow \kappa_{\mathfrak{m}} \in \mathcal{O}_{(\mathfrak{l})}^{*} / \mathcal{O}_{(\mathfrak{l})}^{* M}
$$

for all prime ideals $\mathfrak{l}$. We call $\kappa_{\mathfrak{m}}$ the Kolyvagin class indexed by $\mathfrak{m}$ associated to the Euler system $\left\{\xi_{\mathfrak{m}}\right\}$.

3.3. Some operators and Kolyvagin recursion. Let $\mathfrak{l}$ be a prime ideal dividing $\mathbf{m}$. Let $\nu_{\mathfrak{l}}: \mathcal{O}_{(\mathfrak{l})}^{*} / \mathcal{O}_{(\mathfrak{l})}^{* M} \rightarrow(\mathcal{O} / \mathfrak{l})_{M}^{*}$ be the unique homomorphism such that

$$
\nu_{\mathfrak{l}}\left(x \bmod \mathcal{O}_{(\mathfrak{l})}^{* M}\right) \equiv x^{(|\mathfrak{l}|-1) / M} \bmod \mathfrak{l} \mathcal{O}_{(\mathfrak{l})}
$$

for all $x \in \mathcal{O}_{(\mathfrak{l})}^{*}$. Let $[\cdot]_{\mathfrak{l}}: F^{*} / F^{* M} \rightarrow \mathcal{C}_{(\mathfrak{l})} \otimes(\mathbb{Z} / M \mathbb{Z})$ be the unique homomorphism such that

$$
\left[x \bmod F^{* M}\right]_{\mathfrak{r}}=x \mathcal{O}_{(\mathfrak{l})} \bmod \left(\mathcal{C}_{(\mathfrak{l})}\right)^{M}
$$

for all $x \in F^{*}$, where $\mathcal{C}_{(\mathfrak{l})}$ is the group of fractional $\mathcal{O}_{(\mathfrak{l})}$-ideals. As in the classical case (cf. $[\mathrm{AO}, \S 2.4]$ ) there exists a unique isomorphism

$$
\exp _{\mathfrak{l}}: \mathcal{C}_{(\mathfrak{l})} \otimes(\mathbb{Z} / M \mathbb{Z}) \rightarrow(\mathcal{O} / \mathfrak{l})_{M}^{*}
$$

such that

$$
\exp _{\mathfrak{l}}\left(x^{N_{\mathfrak{l}}} \mathcal{O}_{(\mathfrak{l})} \otimes(1 \bmod M)\right) \equiv\left(x^{1-\sigma_{\mathfrak{l}}}\right)^{(|\mathfrak{l}|-1) / M} \bmod \sqrt{\mathfrak{l O}_{\mathfrak{l},(\mathfrak{l})}}
$$

for all $x \in F_{\mathfrak{l}}^{*}$. Then, for all integral ideals $\mathfrak{m}$ dividing $\mathbf{m}$ and prime ideals $\mathfrak{l}$ dividing $\mathfrak{m}$,

$$
\exp _{\mathfrak{l}}\left[\kappa_{\mathfrak{m}}\right]_{\mathfrak{l}} \equiv \nu_{\mathfrak{l}} \kappa_{\mathfrak{m} \mathfrak{l}^{-1}} \bmod \sqrt{\mathfrak{l O} \mathcal{O}_{\mathfrak{m},(\mathfrak{l})}} .
$$

We say that a system of classes $\left\{\varepsilon_{\mathfrak{m}} \in F^{*} / F^{* M}\right\}_{\mathfrak{m} \mid \mathbf{m}}$ satisfies the Kolyvagin recursion if, for all integral ideals $\mathfrak{m}$ dividing $\mathbf{m}$ and prime ideals $\mathfrak{l}$, the following hold:

- $\mathfrak{m} \neq \mathfrak{e},(\mathfrak{l}, \mathfrak{m})=\mathfrak{e} \Rightarrow \varepsilon_{\mathfrak{m}} \in \mathcal{O}_{(\mathfrak{l})}^{*} / \mathcal{O}_{(\mathfrak{l})}^{* M}$.

- $\mathfrak{l} \mid \mathfrak{m} \Rightarrow \exp _{\mathfrak{r}}\left[\varepsilon_{\mathfrak{m}}\right]_{\mathfrak{l}}=\nu_{\mathfrak{l}} \varepsilon_{\mathfrak{m} \mathfrak{l}}-1$.

Proposition 3.3.1 ([AO, Proposition 3.9]). Let $\xi: \mathbb{U}_{\mathbf{m}}^{1} \rightarrow \mathcal{O}_{\mathbf{m}}^{*}$ be any $H_{\mathbf{m}}$-equivariant homomorphism such that $\xi I_{\mathfrak{l}} \subset 1+\sqrt{\mathfrak{I O}_{\mathbf{m},(\mathfrak{l})}}$ for all prime ideals $\mathfrak{l}$ dividing $\mathbf{m}$. Let

$$
\kappa: H^{0}\left(H_{\mathbf{m}}, \mathbb{U}_{\mathbf{m}}^{1} / M \mathbb{U}_{\mathbf{m}}^{1}\right) \rightarrow H^{0}\left(H_{\mathbf{m}}, F_{\mathbf{m}}^{*} / F_{\mathbf{m}}^{* M}\right)=F^{*} / F^{* M}
$$


be the homomorphism induced by $\xi$. Let $\left\{c_{\mathfrak{m}} \in H^{0}\left(H_{\mathfrak{m}}, \mathbb{U}_{\mathfrak{m}}^{1} / M \mathbb{U}_{\mathfrak{m}}^{1}\right)\right\}_{\mathfrak{m} \mid \mathbf{m}}$ be any system of classes satisfying the universal Kolyvagin recursion. Then the system $\left\{\kappa c_{\mathfrak{m}} \in F^{*} / F^{* M}\right\}_{\mathfrak{m} \mid \mathbf{m}}$ also satisfies the Kolyvagin recursion.

Note that, for the $H_{\mathbf{m}}$-equivariant map $\xi, \xi\left(x_{\mathfrak{m}}\right) \equiv \xi\left(x_{\mathfrak{m}}\right)$ modulo primes over $\mathfrak{l}$ is equivalent to $\xi I_{\mathfrak{l}} \subset 1+\sqrt{\mathfrak{l O}_{\mathbf{m},(\mathfrak{l})}}$. We see that an Euler system $\left\{\xi_{\mathfrak{m}}\right\}$ and its Kolyvagin classes $\left\{\kappa_{\mathfrak{m}}\right\}$ can be recovered from the universal Euler system $\left\{x_{\mathfrak{m}}\right\}$ and the universal Kolyvagin classes $\left\{c_{\mathfrak{m}}\right\}$ by the map $\xi$.

\section{Circular distribution}

4.1. Circular distribution. Let $\widetilde{K}:=\bigcup_{\mathfrak{m}} K_{\mathfrak{m}}$ and $\bar{T}^{o}:=\bar{T} \backslash \bar{T}_{\mathfrak{e}}$. A $G$ equivariant map $f: \bar{T}^{o} \rightarrow \widetilde{K}^{*}$ is called a circular distribution on $k$ if, for any $a \in \bar{T}^{o}$ and $\mathfrak{n} \in T_{\mathfrak{e}}$,

$$
\prod_{\mathfrak{n} b=a} f(b)=f(a) .
$$

From the $G$-equivariance we see that $f(a) \in K_{\mathfrak{m}}$ for any $a \in \bar{T}_{\mathfrak{m}}^{o}$. Let $\mathcal{C}$ be the set of all circular distributions on $k$. Then $\mathcal{C}$ becomes an $R=\mathbb{Z}[G]$-module via

$$
(r \cdot f)(a)=\prod \sigma(f(a))^{n_{\sigma}}
$$

where $r=\sum n_{\sigma} \sigma$. Denote by $N_{\mathfrak{m}, \mathfrak{n}}$ the norm map from $K_{\mathfrak{m}}$ to $K_{\mathfrak{n}}$ for $\mathfrak{n} \mid \mathfrak{m}$.

Proposition 4.1.1 ([S1, Theorem 2.1]). Let $f: \bar{T}^{o} \rightarrow \widetilde{K}$ be a $G$ equivariant map. Then the condition (4.1.1) is equivalent to the following two conditions:

- For any prime ideal $\mathfrak{p}$ of $\mathbb{A}$ and a square-free ideal $\mathfrak{m} \neq \mathfrak{e}$ with $\mathfrak{p} \nmid \mathfrak{m}$,

$$
N_{\mathfrak{p m}, \mathfrak{m}} f\left(\left\{\mathfrak{p}^{-1} \mathfrak{m}^{-1}\right\}\right)=f\left(\left\{\mathfrak{m}^{-1}\right\}\right)^{1-\mathrm{Fr}_{\mathfrak{p}}^{-1}}
$$

- For $n-i \geq 1$,

$$
N_{\mathfrak{p}^{n} \mathfrak{m}, \mathfrak{p}^{n-i} \mathfrak{m}} f\left(\left\{\mathfrak{p}^{-n} \mathfrak{m}^{-1}\right\}\right)=f\left(\left\{\mathfrak{p}^{i-n} \mathfrak{m}^{-1}\right\}\right)
$$

Lemma 4.1.2. Let $\mathfrak{p}$ and $\mathfrak{l}$ be two distinct prime ideals of $\mathbb{A}$. Let $\mathcal{L}_{u}$ be a prime ideal of $K_{p^{p}}$ lying over $\mathfrak{l}$. Then the decomposition group of $\mathcal{L}_{u+1}$ in $K_{\mathfrak{p}^{p^{u+1}}} / K_{\mathfrak{p}^{p^{u}}}$ is nontrivial for any sufficiently large $u$, where $p$ is the characteristic of $k$.

Proof. Let $d_{u}$ be the inertial degree of $\mathcal{L}_{u}$ in $K_{\mathfrak{p}^{p^{u}}} / K_{\mathfrak{e}}$. Let $x \in \mathbb{A}$ be such that

$$
N_{K_{\mathfrak{e}} / k} \mathcal{L}_{0}=(x), \quad \operatorname{sgn}(x)=1 .
$$

Then from $[\mathrm{H} 1, \S 4], d_{u}$ is the order of $x$ in $\left(\mathbb{A} / \mathfrak{p}^{p^{u}}\right)^{*}$. Write $x^{d_{1}}=1+z$, where $z \in \mathfrak{p}^{m} \backslash \mathfrak{p}^{m+1}$. Let $t$ be such that $p^{t} \leq m<p^{t+1}$. Then $d_{t}=d_{1}$. Let $u>t$. Then it is not hard to see that $d_{u}=d_{t} p^{u-t}$. Hence the result follows. 
Lemma 4.1.3. Let $f \in \mathcal{C}$ and $\mathfrak{p}$ be a prime ideal of $\mathbb{A}$. If $\mathfrak{m}$ is divisible by at least two distinct prime ideals (resp. a $\mathfrak{p}$-power), then $f\left(\left\{\mathfrak{m}^{-1}\right\}\right)$ is a unit (resp. a p-unit).

Proof. Let $\mathcal{L}_{u}$ be a prime ideal dividing $f\left(\left\{\mathfrak{p}^{-p^{u}}\right\}\right)$ in $K_{\mathfrak{p}^{u}}$, which is prime to any prime ideal above $\mathfrak{p}$. The condition (4.1.3) and Lemma 4.1.2 imply that the principal ideal $\left(f\left(\left\{\mathfrak{p}^{-p^{u}}\right\}\right)\right)$ is divisible by any sufficiently large power of $\mathcal{L}_{u}$, which is a contradiction. Hence $f\left(\left\{\mathfrak{p}^{-p^{u}}\right\}\right)$ is a $\mathfrak{p}$-unit. Again by (4.1.3), $f\left(\mathfrak{p}^{-s}\right)$ is a $\mathfrak{p}$-unit for any positive integer $s$. A similar argument implies that $f\left(\left\{\mathfrak{m}^{-1}\right\}\right)$ is a unit if $\mathfrak{m}$ is divisible by at least two distinct prime ideals.

Let $\mathcal{F}$ be an $R$-submodule of $\mathcal{C}$ consisting of $f \in \mathcal{C}$ such that for any nonunit integral ideal $\mathfrak{m}$ and any prime ideal $\mathfrak{l}$ with $\mathfrak{l} \nmid \mathfrak{m}$,

$$
f\left(\left\{\mathfrak{l}^{-1} \mathfrak{m}^{-1}\right\}\right) \equiv f\left(\left\{\mathfrak{m}^{-1}\right\}\right)^{\mathrm{Fr}_{\mathfrak{l}}^{-1}} \bmod \mathcal{L}
$$

where $\mathcal{L}$ is any prime ideal above $\mathfrak{l}$.

Let $\psi: \bar{T}^{o} \rightarrow \widetilde{K}^{*}$ be the $G$-equivariant map defined by

$$
\psi\left(\left\{\mathfrak{m}^{-1}\right\}\right)=\lambda_{\mathfrak{m}}=\xi(\mathfrak{m}) e_{\mathfrak{m}}(1),
$$

where $\xi(\mathfrak{a})$ is the $\xi$-invariant of $\mathfrak{a}$ and $e_{\mathfrak{a}}$ is the Drinfeld lattice function associated to a. See [H2] for details. From Theorem 5.1 of [H1],

$$
\psi\left(\left\{\mathfrak{m}^{-1}\right\}\right)=\varrho_{\mathfrak{l}}^{(\mathfrak{l m})}\left(\psi\left(\left\{\mathfrak{l}^{-1} \mathfrak{m}^{-1}\right\}\right)\right) \equiv \psi\left(\left\{\mathfrak{l}^{-1} \mathfrak{m}^{-1}\right\}\right)^{q^{\operatorname{deg} \mathfrak{l}}} \bmod \mathcal{L},
$$

which implies that $\psi \in \mathcal{F}$. As in the classical case one can state an analogue of Coleman's conjecture, that is, $\mathcal{F}=R \psi$.

4.2. Torsions in circular distribution. In this section we describe the torsion subgroups of $\mathcal{C}$ and $\mathcal{F}$. Let $S$ be any nonempty set of square-free integral ideals. Let $\delta_{S}$ be a $G$-equivariant map on $\bar{T}^{o}$ defined by

$$
\delta_{S}\left(\left\{\mathfrak{m}^{-1}\right\}\right)= \begin{cases}\gamma & \text { if there is } \mathfrak{s} \in S \text { such that } \\ \mathfrak{m} \text { and } \mathfrak{s} \text { have the same prime factors } \\ 1 \quad \text { otherwise, }\end{cases}
$$

where $\gamma$ is a fixed generator of $\mathbb{F}_{q}^{*}$. Then it is easy to see that $\delta_{S} \notin \mathcal{F}$, unless $S$ is the set of all square-free integral ideals. Let $J \subset H$ be the sign group, which is isomorphic to $\mathbb{F}_{q}^{*}$. Let $\mathcal{D}$ be the $R$-submodule of $\mathcal{C}$ generated by $\delta_{S}$ for all $S$ as above.

Lemma 4.2.1. $\mathcal{D}$ is the submodule of $\mathcal{C}$ consisting of all elements $f$ such that $f^{q-1}=1$, that is, the values of $f$ lie in $\mathbb{F}_{q}^{*}$.

Proof. Suppose that $f^{q-1}=1$. For each $i$ with $0<i<q-1$, let

$$
S_{i}=\left\{\mathfrak{m}: \mathfrak{m} \text { square free and } f\left(\left\{\mathfrak{m}^{-1}\right\}\right)=\gamma^{i}\right\} .
$$


Then we can easily see that

$$
f=\prod_{i} \delta_{S_{i}}^{i}
$$

One can follow [S2, §3] to obtain

Proposition 4.2.2. Let $f \in \mathcal{F}$. Then $\left\{f\left(\left\{\mathfrak{p}_{1}^{-n_{1}} \cdots \mathfrak{p}_{r}^{-n_{r}}\right\}\right)\right\}_{n_{i} \in \mathbb{N}} \nsubseteq \mathbb{F}_{q}^{*}$ if and only if

$$
\lim _{n}\left[K_{\mathfrak{e}}\left(f\left(\left\{\mathfrak{p}_{1}^{-u_{1}} \cdots \mathfrak{p}_{i}^{-n} \cdots \mathfrak{p}_{r}^{-u_{r}}\right\}\right)\right): K_{\mathfrak{e}}\right]=\infty \quad \text { for some } i .
$$

Moreover, if $f\left(\left\{\mathfrak{p}_{1}^{-u_{1}} \cdots \mathfrak{p}_{i}^{-n} \cdots \mathfrak{p}_{r}^{-u_{r}}\right\}\right) \notin \mathbb{F}_{q}^{*}$ for all $n$, then

$$
\left[K_{\mathfrak{e}}\left(f\left(\left\{\mathfrak{p}_{1}^{-u_{1}} \cdots \mathfrak{p}_{i}^{-n-1} \cdots \mathfrak{p}_{r}^{-u_{r}}\right\}\right)\right): K_{\mathfrak{e}}\left(f\left(\left\{\mathfrak{p}_{1}^{-u_{1}} \cdots \mathfrak{p}_{i}^{-n} \cdots \mathfrak{p}_{r}^{-u_{r}}\right\}\right)\right)\right] \geq p
$$

for all sufficiently large $n$.

Corollary 4.2.3. $\mathcal{C}_{\text {tor }}=\mathcal{D}$.

Let $\delta$ be a $G$-equivariant function on $\bar{T}^{o}$ defined by $\delta\left(\left\{\mathfrak{m}^{-1}\right\}\right)=\gamma$.

Proposition 4.2.4. $\mathcal{F}_{\text {tor }}=\langle\delta\rangle$.

Proof. Let $1 \neq f \in \mathcal{F}_{\text {tor. }}$ Then $f=\prod_{i=1}^{q-2} \delta_{S_{i}}^{i}$. Then $S_{i} \neq \emptyset$ for some $i$. Let $\mathfrak{n} \in S_{i}$ and $\mathfrak{p}$ be a prime ideal not dividing $\mathfrak{n}$. Then

$$
\gamma^{i}=f\left(\left\{\mathfrak{n}^{-1}\right\}\right) \equiv f\left(\left\{\mathfrak{p}^{-1} \mathfrak{n}^{-1}\right\}\right) \bmod \mathcal{P} .
$$

Since $q>2, \mathfrak{p n} \in S_{i}$. In this way we see that every square-free integral ideal divisible by $\mathfrak{n}$ lies in $S_{i}$. Now processing backwards we see that every square-free integral ideal lies in $S_{i}$, which implies the result.

REMark 4.2.5. One easily sees that $\delta=\psi^{\sigma_{\gamma}-1}$, where $\sigma_{\gamma}\left(\lambda_{\mathfrak{m}}\right)=\gamma \lambda_{\mathfrak{m}}$. But in the classical case, the circular distribution $\delta_{\text {odd }}$ in [S2], defined by $\delta_{\text {odd }}\left(\zeta_{m}\right)= \pm 1$ depending on whether $m$ is even or odd, is not contained in $R \psi$, as we now prove.

Proposition 4.2.6. With the notations as in [S2, §3], we have

$$
\delta_{\text {odd }} \notin R \psi \text {. }
$$

Proof. Suppose that $\delta_{\text {odd }}=r \cdot \psi$ for some $r \in R$. On $\mathbb{Q}\left(\zeta_{12}\right)$ we can write

$$
r=n_{1} \mathrm{id}+n_{\sigma} \sigma+n_{\tau} \tau+n_{\sigma \tau} \sigma \tau,
$$

where $\sigma(i)=i, \sigma\left(\zeta_{3}\right)=\zeta_{3}^{-1}, \tau(i)=-i$ and $\tau\left(\zeta_{3}\right)=\zeta_{3}$. Since $\psi(\zeta)=1-\zeta$,

$$
\begin{aligned}
-1 & =r \cdot \psi\left(\zeta_{3}\right)=\left(-\zeta_{3}\right)^{n_{\sigma}+n_{\sigma \tau}}\left(1-\zeta_{3}\right)^{n_{1}+n_{\sigma}+n_{\tau}+n_{\sigma \tau}}, \\
1 & =r \cdot \psi\left(i \zeta_{3}\right)=(i)^{n_{\sigma}}\left(-\zeta_{3}\right)^{n_{\tau}}\left(i \zeta_{3}^{-1}\right)^{n_{\sigma \tau}}\left(1-i \zeta_{3}\right)^{n_{1}+n_{\sigma \tau}-n_{\sigma}-n_{\tau}} .
\end{aligned}
$$

From (1), $n_{\sigma}+n_{\sigma \tau}$ must be odd, but from (2) it must be even, which is a contradiction.

Therefore Coleman's conjecture in the classical case should be $\mathcal{F}_{\mathrm{f}}=R \psi$, where $\mathcal{F}_{\mathrm{f}}$ is the free part of $\mathcal{F}$. 
4.3. Circular distributions and Euler systems. Let $\mathcal{E}_{F}$ be the set of all Euler systems on $F$. Let $C_{F}$ be the group of cyclotomic units in the sense of $[\mathrm{ABJ}]$.

Let $f \in \mathcal{F}$ and $\mathfrak{t}$ be a nonunit integral ideal prime to $\mathbf{m}$. For $\mathfrak{m} \mid \mathbf{m}$, define

$$
\alpha(f, \mathfrak{t})_{\mathfrak{m}}=N_{K_{\mathfrak{m}[f, \mathfrak{t}]} / F_{\mathfrak{m}}} f\left(x_{\mathfrak{m} \mathfrak{t}}\right),
$$

where $[\mathfrak{f}, \mathfrak{t}]$ denotes the least common multiple of $\mathfrak{f}$ and $\mathfrak{t}$. Then we have the following lemma as in the classical case ([S1, Lemma 4.1]).

LEMma 4.3.1. $\left\{\alpha(f, \mathfrak{t})_{\mathfrak{m}}\right\}_{\mathfrak{m} \mid \mathbf{m}}$ is an Euler system.

REMARK 4.3.2. (a) The examples in $\S 3.2$ are obtained from $\alpha(\psi, \mathfrak{t})$ for some appropriate $\mathfrak{t}$.

(b) In the classical case, for $\mathfrak{t}$ prime to the conductor of $F, \alpha(f, \mathfrak{t})$ does not give any new units of $F$, so this case was not considered in [S1]. But in the global function field case, elliptic units in the sense of Hayes ([H2]) can be obtained in this way.

Let $\widehat{\mathcal{E}}_{F}(\mathfrak{m})$ (resp. $\left.\widehat{\mathcal{F}}_{F}(\mathfrak{m})\right)$ be the $G_{\mathfrak{m}}$-submodule generated by the elements $\xi_{\mathfrak{n}}$ (resp. $\left.\alpha(f, \mathfrak{t})_{\mathfrak{n}}\right)$ for $\mathfrak{n} \mid \mathfrak{m}$ with $\left\{\xi_{\mathfrak{n}}\right\} \in \mathcal{E}_{F}$ (resp. $f \in \mathcal{F}$ and $\mathfrak{t} \neq \mathfrak{e}$ prime to $\mathbf{m}$ ). Let $E_{F}(\mathfrak{m})$ (resp. $C_{F}(\mathfrak{m})$ ) be the group of global (resp. cyclotomic) units in $F_{\mathfrak{m}}$. Let

$$
\mathcal{E}_{F}(\mathfrak{m})=\widehat{\mathcal{E}}_{F}(\mathfrak{m}) \cap E_{F}(\mathfrak{m}), \quad \mathcal{F}_{F}(\mathfrak{m})=\widehat{\mathcal{F}}_{F}(\mathfrak{m}) \cap E_{F}(\mathfrak{m}) .
$$

It is shown in [ABJ, Theorem 3.9 and 5.1] that if $\ell$ does not divide $q(q-1)\left[F_{\mathfrak{m}}: k\right]$, then the $\ell$-class number of $F_{\mathfrak{m}}^{+}$is equal to the $\ell$-primary part of $E_{F_{\mathrm{m}}^{+}} / C_{F_{\mathrm{m}}^{+}}$. Then following the same argument as in $\S 4$ of [S1], we have

TheOREM 4.3.3. Let $\ell$ be a prime number as above and $\mathfrak{m} \mid \mathbf{m}$. Then

$$
\mathcal{E}_{F}(\mathfrak{m}) \otimes \mathbb{Z}_{\ell}=\mathcal{F}_{F}(\mathfrak{m}) \otimes \mathbb{Z}_{\ell}=C_{F}(\mathfrak{m}) \otimes \mathbb{Z}_{\ell}
$$

Acknowledgments. The authors would like to thank the referee for many valuable comments on the paper.

\section{References}

[ABJ] J. Ahn, S. Bae and H. Jung, Cyclotomic units and Stickelberger ideals of global function fields, Trans. Amer. Math. Soc. 355 (2003), 1803-1818.

[A] G. Anderson, A double complex for computing the sign-cohomology of the universal ordinary distribution, in: Contemp. Math. 224, Amer. Math. Soc., 1999, 1-27.

[AO] G. Anderson and Y. Ouyang, A note on cyclotomic Euler systems and the double complex method, Canad. J. Math. 55 (2003), 673-692.

[BK] S. Bae and P.-L. Kang, Double complex and gamma monomials in global function fields, J. Number Theory 109 (2004), 69-88.

[H1] D. Hayes, Stickelberger elements in function fields, Compos. Math. 55 (1985), 209-235. 
[H2] D. Hayes, Elliptic units in function fields, in: Number Theory Related to Fermat's Last Theorem, N. Koblitz (ed.), Progr. Math. 26, Birkhäuser, 1982, 321-340.

$[\mathrm{Ku}] \quad$ D. Kubert, The universal ordinary distribution, Bull. Soc. Math. France 107 (1979), 179-202.

[O] Y. Ouyang, Group cohomology of the universal ordinary distribution, J. Reine Angew. Math. 537 (2001), 1-32.

[S1] S. Seo, Circular distribution and Euler systems, J. Number Theory 88 (2001), 366-379.

[S2] -, A note on circular distributions, Acta Arith. 114 (2004), 313-322.

[XZ] F. Xu and J. Q. Zhao, Euler systems in global function fields, Israel J. Math. 124 (2001), 367-379.

[Y] L. S. Yin, Distributions on a global fields, J. Number Theory 80 (2000), 154-167.

Department of Mathematics

KAIST

Taejon 305-701, Korea

E-mail: shbae@math.kaist.ac.kr
Department of Mathematical Sciences

Tsinghua University Beijing 100084, P.R. China E-mail: lsyin@math.tsinghua.edu.cn

Received on 30.3.2005

and in revised form on 16.1.2007 